\title{
Análise isotópica de oxigênio e carbono em microfósseis da Formação Romualdo, cretáceo inferior, bacia do Araripe, Pernambuco, nordeste do Brasil
}

Rilda Verônica Cardoso de Araripe ${ }^{1 *}$, David Holanda de Oliveira ${ }^{2}$, Maria Emília Travassos Rios Tomé ${ }^{3}$, Renata Moura de Mello ${ }^{4}$ Alcina Magnólia Franca Barreto ${ }^{1}$ Alcides Nóbrega Sial ${ }^{3}$

${ }^{1}$ Programa de Pós-Graduação em Geociências Departmento de Geologia Universidade Federal de Pernambuco Recife PE Brazil CEP 50670-901

${ }^{2}$ Centro de Ciências Agrárias - Campus III Departamento de Ciências Biológicas Centro de Ciências Agrárias Universidade Federal da Paraíba Centro, Areia PB Brazil CEP 58397-000

${ }^{3}$ Departamento de Geologia

Laboratório de Isótopos Estáveis (NEG-LABISE) Universidade Federal de Pernambuco Recife PE Brazil

CEP 50670-901

${ }^{4}$ PETROBRAS - CENPES Av. Horácio de Macedo 950 llha do Fundão,

Rio de Janeiro RJ Brazil, CEP 21941-915

* Autora Correspondente, rildacardoso@gmail.com Tel.: +55 (81) 2126-8240

\section{RESUMO}

$\mathrm{O}$ presente trabalho examinou o comportamento de isótopos de $\mathrm{O}$ e $\mathrm{C}$ em carapaças de ostracodes e foraminíferos da Formação Romualdo, Bacia do Araripe - PE. As amostras analisadas são de afloramentos (Arrojado, Canastra e Cedro) localizados nos municípios de Exu e Araripina, nas porções centro-sul e sudoeste da bacia. Os valores de $\delta^{18} \mathrm{O}$ apresentaram variação entre $-10,1 \%$ a $-10,2 \%$ VPDB, e os valores de $\delta^{13} \mathrm{C}$ variaram entre $-12,6 \%$ e $+2,7 \%$ VPDB. Os resultados encontrados para os dados isotópicos de oxigênio indicam águas de temperatura elevada e origem marinha para os calcários. Para os dados isotópicos de carbono nos afloramentos Arrojado e Canastra, os valores indicam ambiente anóxico e com alto teor de matéria orgânica. No afloramento Cedro, ocorre uma variação positiva indicando um aumento na produção primária, também associada a um evento anóxico, sendo os valores positivos interpretados como um pulso marinho, ou seja, o afloramento cedro localizado na porção centro-sul da bacia possui características ligadas a ambiente com maior influência marinha do que nos afloramentos Arrojado e Canastra, localizados na porção sudoeste.

Palavras-Chave: foraminíferos, ostracodes, isótopos estáveis, influencia marinha, Cretáceo.

\section{ABSTRACT}

This work examined the behavior of $\mathrm{C}$ and $\mathrm{O}$ isotope in carapaces of ostracodes and foraminifera from the Romualdo Formation, Araripe Basin - PE. The analyzed samples were collected from three outcrops (Arrojado, Canastra and Cedro) located in the cities of Exu and Araripina. The de $\delta^{18} \mathrm{O}$ values ranged from $-10.1 \%$ to $-10.2 \%$ VPDB and the $\delta^{13} \mathrm{C}$ values ranged from $-12.6 \%$ to $+2.7 \%$ VPDB. Oxygen stabe isotope data indicate high temperature of waters and the deposition of marine limestones in all outcrops. For Arrojado and Canastra outcrops, $\delta^{13} \mathrm{C}$ values indicate an anoxic environment with high content of organic matter. In Cedro outcrop, a positive variation occurs indicating an increase in primary production, also associated to an anoxic event, and the positive values are interpreted as a marine pulse. These results indicate that Cedro outcrop, located in the midsouthern portion of this basin, has environmental characteristics with greater marine influence than in the Arrojado and Canastra outcrops, located in the southwestern portion of this basin.

Keywords: Foraminifera, ostracods, stable isotopes, marine influence, Cretaceous. 


\section{INTRODUÇÃO}

A Bacia Sedimentar do Araripe é a única bacia interior do Nordeste do Brasil que guarda o registro da transgressão marinha durante o Cretáceo Inferior (Ponte, 1992). A Formação Romualdo, corresponde a rochas sedimentares de ambiente transicional a marinho raso, depositadas durante o Neoaptiano-Eoalbiano. Tal formação é caracterizada por ampla gama de litologias (arenitos calcíferos, calcários, margas, folhelhos, calcários coquinoides) embora haja discussões em relação aos níveis estratigráficos com fácies propriamente marinha (Assine et al, 2014).

Os microfóssies fornecem uma riqueza de informações paleoambientais que auxiliam no reconhecimento de sistemas deposicionais, que se desenvolveram em resposta a mudanças do nível relativo do mar. Alguns microfósseis carbonáticos, como ostracodes e foraminíferos, são utilizados como proxies permitindo inferências de parâmetros que auxiliam em tais reconstruções paleoambientais. (Leckie e Olson, 2012).

Os foraminíferos e ostracodes são bastante utilizados em estudos geoquímicos, podendo preservar em sua carapaça valores isotópicos por milhões de anos. Alguns fatores como diagenética, efeito vital $\mathrm{e}$ as tolerâncias ecológicas de cada espécie são fatores limitantes que podem alterar os valores isotópicos, porém tais microfósseis carbonáticos são um dos principais indicadores

\section{1. ÁREA DE ESTUDO}

A Bacia do Araripe esta localizada entre os meridianos $38^{\circ} 30^{\prime}$ e $40^{\circ} 50^{\prime}$ de longitude oeste e os paralelos $7^{\circ} 05^{\prime}$ e $7^{\circ} 50^{\prime}$ de longitude sul (Viana e Neumann, 2002). Sua área de extensão não se limita à Chapada do Araripe, estendendose também pelo Vale do Cariri, num total de aproximadamente $9.000 \mathrm{~km}^{2}$, sendo a mais extensa das bacias interiores do Nordeste do Brasil. Tal bacia é formada por unidades das seqüências pré-rift, rift, pós-rift I e pós-rift II. (Assine, 1992; 2007). A Formação Romualdo, é inserida na sequência pós-rift I (Aptiano/Cenomaniano).

As coletas foram feitas com controle estratigráfico em três afloramentos, nos sítios Arrojado e Canastra, em Araripina e Cedro, em Exu, Pernambuco, regiões centro-sul e sudoeste da Bacia do Araripe (Figura 01).

$\mathrm{O}$ afloramento Arrojado, localizado em Araripina apresenta espessura de $11 \mathrm{~m}$. Na base, da composição da água do mar no passado. As proporções isotópicas na carapaça de foraminíferos e ostracodes podem ser determinadas individualmente ou combinando números relativamente pequenos de carapaças das mesmas espécies, sendo uma vantagem da utilização desses dois grupos para o fracionamento isotópico (Holmes, 1996).

Os estudos das razões de $\delta^{13} \mathrm{C}$ e de $\delta^{18} \mathrm{O}$ são utilizados como traçadores geoquímicos (proxies) e permitem inferências paleoambientais. $\quad \mathrm{O} \quad \delta^{18} \mathrm{O}$ depende principalmente da relação isotópica da água em que é precipitada, da temperatura de calcificação e, em menor grau, da concentração de íons de carbonato, podendo trazer informações sobre a composição da água e condições ambientais, como temperatura e salinidade, em que foi precipitada (Pearson, 2012). Já os valores de $\delta^{13} \mathrm{C}$ são controlados pela proporção de carbono inorgânico dissolvido na água e processos fisiológicos, tais como respiração e fotossíntese, trazendo inferências sobre produção primária sendo importantes na detecção de períodos de anoxia (Spero et al, 1997).

O presente trabalho tem como objetivo contribuir para as interpretações paleoambientais através de análise das razões isotópicas de $\mathrm{C}$ e $\mathrm{O}$ em carapaças de foraminíferos e ostracodes da Formação Romualdo.

é observado o contato do folhelho da Formação Romualdo com rochas do embasamento cristalino. Há um predomínio de camadas de arenito calcífero intercalados aos folhelhos. São encontrados foraminíferos bentônicos no topo da seção, e níveis com abundância de ostracodes mixohialinos na base.

O afloramento Canastra, também localizado em Araripina, é caracterizado por uma seção de quatro metros, tendo na base, folhelhos com concreções carbonáticas, intercalados a arenitos calcíferos, que exibem estratificação cruzada de pequeno porte e, na porção superior, arenitos calcíferos. Assim como no afloramento Arrojado, são encontrados foraminíferos bentônicos no topo da seção, e níveis com abundância de ostracodes mixohialinos na base.

O afloramento Cedro encontra-se próximo a cidade de Exu possui cerca de seis metros de espessura, sendo os primeiros três metros 
compostos de camadas de folhelho com intercalações de arenito com base erosional e estruturas de sobrecarga, seguido de uma fina camada de arenito calcífero. O topo da seção é composto por intercalações de siltito seguido de 10 camadas de arenito calcífero. Nesse afloramento é encontrada uma diversidade de foraminíferos bentônicos ao longo da seção, e a ocorrência de foraminíferos planctônicos em alguns níveis (1,3 e 1,9 m). A associação de microfósseis encontrada nesse afloramento é composta por foraminíferos e ostracodes, presentes nos mesmos níveis.

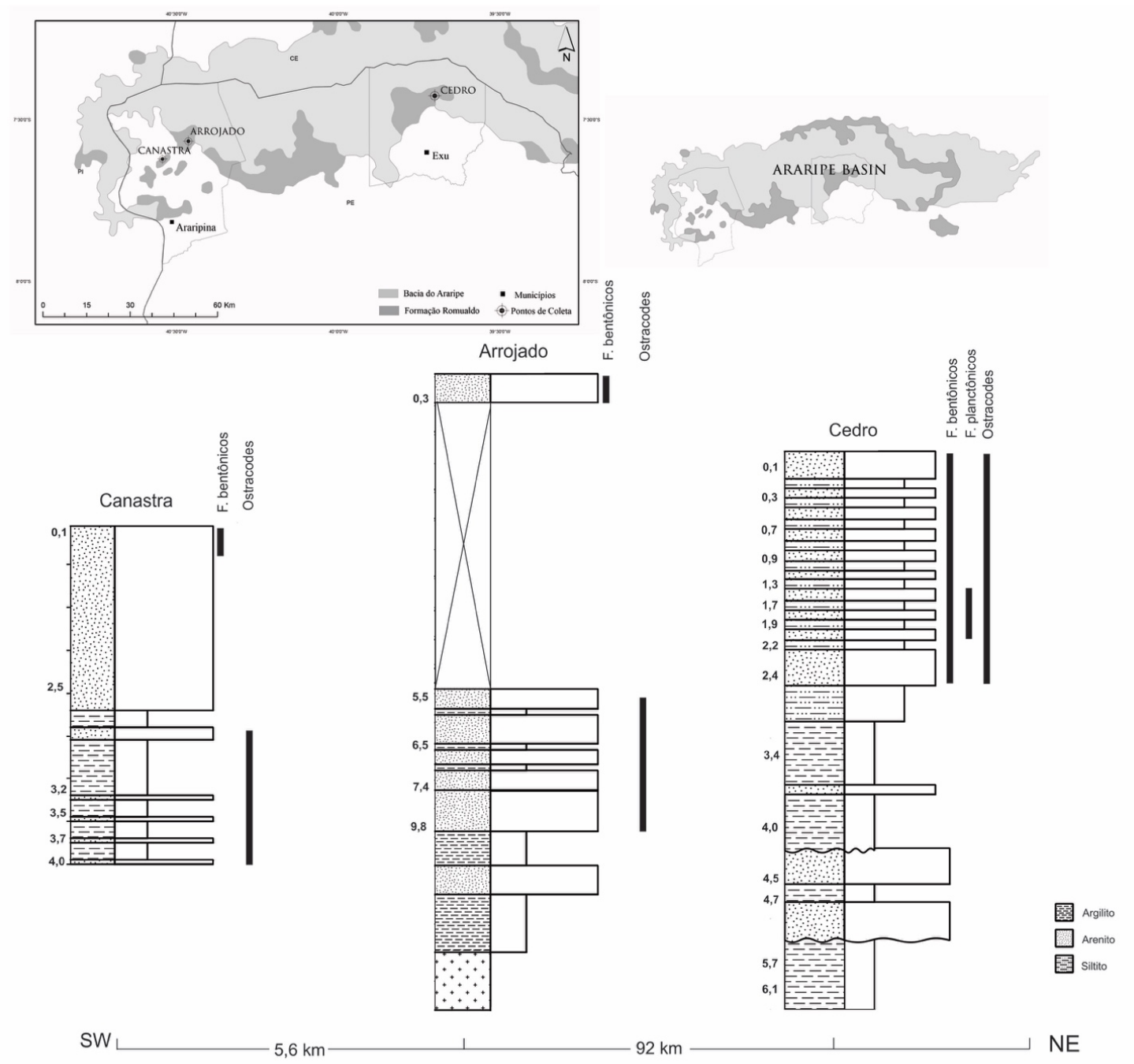

Figura 1

Localização dos afloramentos na Bacia do Araripe, municípios de Exu e Araripina. Perfis estratigráficos dos afloramentos com a distribuição dos microfósseis ao longo da seção.

\section{MATERIAL E MÉTODOS}

O estudo das associações de ostracodes e foraminíferos foram realizados em 18 amostras, provenientes de três seções estratigráficas, sendo Cedro (Exu), localizado na porção centro-sul da bacia, e Arrojado e Canastra, ambos em Araripina, sudoeste da bacia. Essas áreas foram selecionadas para estudo por conter concentrações de fósseis de invertebrados (equinoides, gastrópodes e biválvios) em bom estado de preservação. Devido à ocorrência de calcarenitos com boa diversidade e preservação de fósseis de moluscos e equinoides cassiduloides.

A preparação, triagem e análise dos microfósseis seguiu a metodologia padrão para microfósseis carbonáticos proposto por Leipnitz \& Aguiar (2002). Os procedimentos foram realizados no Laboratório de Paleontologia do Departamento de Geologia da UFPE (PALEOLAB).

As análises isotópicas das amostras foram realizadas no NEG-LABISE - UFPE. Os microfósseis após triagem e separação foram 
pulverizados. O NEG- LABISE utiliza o método convencional de digestão (McCrea, 1950). Para os valores isotópicos de oxigênio e carbono, o pó foi diluído com ácido ortofosfórico a $25^{\circ} \mathrm{C}$ para liberar $\mathrm{CO}_{2}$. Os valores de $\delta^{13} \mathrm{C}$ e $\delta^{18} \mathrm{O}$ foram medidos em $\mathrm{CO}_{2}$. Os dados isotópicos foram calibrados com padrões internacionais e os resultados

\section{RESULTADOS E DISCUSSÃO}

Das 18 amostras colocadas para análise isotópica de $\delta^{18} \mathrm{O}$ e $\delta^{13} \mathrm{C}$ em carapaça de foraminíferos, apenas em 6 amostras foi obtido resultados (Tabela 1). Os valores da composição isotópica de $\delta^{18} \mathrm{O}$ em carapaças de foraminíferos apresentaram valores entre $10,08 \%$ a $-10,15 \%$, não havendo grandes oscilações. As análises isotópicas do $\delta^{13} \mathrm{C}$ apresentados em V-PDB (Vienna Pee Dee Belemnite).

A análise isotópica foi extraída da carapaça de ostracodes e foraminíferos. As espécies utilizadas para análise foram Pattersoncypris symmetrica e Agathammina sp. que se apresentaram mais frequentes, ocorrendo em todos os afloramentos.

variaram entre valores negativos de $-6,24 \%$ o 1,19\%. Devido ao baixo número de amostras que apresentaram resultados e a baixa quantidade de carapaças de foraminíferos para novas análises, foram considerados apenas os dados isotópicos de $\delta^{18} \mathrm{O}$ e $\delta^{13} \mathrm{C}$ realizados em carapaça de ostracodes.

Tabela 1 - Resultados das análises isotópicas de oxigênio e carbono em carapaças de foraminíferos provenientes dos afloramentos Cedro, Arrojado e Canastra.

\begin{tabular}{|c|c|c|c|}
\hline & Prof. (m) & $\delta^{13} C_{\text {VPDB }} \%$ & $\delta^{18} O_{\text {VPDB }} \%$ \\
\hline \multirow{4}{*}{ 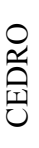 } & 0,1 & $-1,19$ & $-10,15$ \\
\hline & 0,7 & $-3,14$ & $-10,11$ \\
\hline & 1,9 & $-5,15$ & $-10,06$ \\
\hline & 2,2 & $-4,91$ & $-10,10$ \\
\hline 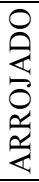 & 0,3 & $-4,88$ & $-10,10$ \\
\hline 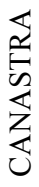 & 3,7 & $-6,24$ & $-10,08$ \\
\hline
\end{tabular}

Os valores da composição isotópica de $\delta^{18} \mathrm{O}$ em carapaças de ostracodes apresentou comportamento bastante homogêneo, todos os afloramentos apresentam valores que oscilaram entre $-10,08 \%$ a $-10,20 \%$. Para os dados de oxigênio, o efeito vital não apresentou grande influência na variação dos dados, devido às analises serem feitas em ostracodes do mesmo gênero. Holser et al. (1996) sugerem que as

\subsection{ANÁLISE DE $\delta^{18} O$}

A composição química da água e a temperatura são consideradas fatores importantes que causam o fracionamento isotópico do oxigênio. Nas águas oceânicas o $\mathrm{O}^{16}$ evapora mais rápido que $\mathrm{o} \mathrm{O}^{18}$, no entanto o $\mathrm{O}^{16}$ (por ser mais leve) também precipita mais que o $\mathrm{O}^{18}$. Os carbonatos precipitados em água morna acabam incorporando mais $\mathrm{O}^{16} \mathrm{e}$ amostragens sejam feitas a nível de espécies ou gêneros e argumentam que as distorções decorrentes do efeito vital são sutis e geralmente não alteram a forma da curva. Os valores da composição isotópica do $\delta^{13} \mathrm{C}$ variaram entre $-12,6 \%$ tendo valor mais negativo no afloramento Canastra, e valor mais positivo a $+2,7 \%$ no afloramento Cedro (Tabela 2). menos $\mathrm{O}^{18}$ (e frequentemente tem um valor de razão $\delta^{18} \mathrm{O}$ mais negativo) que aqueles precipitados em águas frias (Rodrigues e Fauth, 2013).

A salinidade é igualmente afetada, em uma escala regional, a água doce tem muito mais $\mathrm{O}^{16}$ que a água do mar. Os carbonatos precipitados em água doce, portanto, tendem a 
incorporar mais $\mathrm{O}^{16}$ e menos $\mathrm{O}^{18}$ (tendo um $\delta^{18} \mathrm{O}$ mais negativo) que aqueles precipitados em água do mar normal. Assim, em ambientes de água salobra, os valores de $\delta^{18} \mathrm{O}$ são geralmente mais baixos que os calcários marinhos normais, devido a assinatura isotópica da água doce (Armstrong \& Brasier, 2005).

De acordo com isso, na figura 2 estão representados os valores de $\delta^{18} \mathrm{O}$ nas seções estudadas, observa-se que os valores se apresentaram bastante negativos e com pouca variação.

Tabela 2 - Resultados das análises isotópicas de oxigênio e carbono em carapaças de ostracodes provenientes dos afloramentos Cedro, Arrojado e Canastra.

\begin{tabular}{|c|c|c|c|}
\hline & Prof. (m) & $\delta^{13} C_{\text {VPDB }} \%$ & $\delta^{18} O_{\text {VPDB }} \%$ \\
\hline \multirow{8}{*}{$\begin{array}{l}\stackrel{0}{0} \\
\text { 穵 }\end{array}$} & 0,1 & $-2,1$ & $-10,11$ \\
\hline & 0,3 & $-1,4$ & $-10,17$ \\
\hline & 0,7 & $-0,4$ & $-10,16$ \\
\hline & 0,9 & $-2,2$ & $-10,12$ \\
\hline & 1,3 & 1,5 & $-10,11$ \\
\hline & 1,9 & 2,7 & $-10,15$ \\
\hline & 2,2 & $-4,2$ & $-10,15$ \\
\hline & 2,4 & $-4,7$ & $-10,13$ \\
\hline \multirow{5}{*}{ 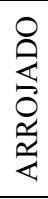 } & 0,3 & $-3,7$ & $-10,20$ \\
\hline & 5,5 & $-5,8$ & $-10,17$ \\
\hline & 6,5 & $-2,7$ & $-10,15$ \\
\hline & 7,4 & $-4,3$ & $-10,17$ \\
\hline & 9,8 & $-7,6$ & $-10,15$ \\
\hline \multirow{5}{*}{ 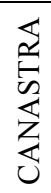 } & 2,5 & $-5,9$ & $-10,09$ \\
\hline & 3,2 & $-12,6$ & $-10,20$ \\
\hline & 3,5 & $-5,3$ & $-10,13$ \\
\hline & 3,7 & $-6,2$ & $-10,08$ \\
\hline & 4 & $-4,5$ & $-10,11$ \\
\hline
\end{tabular}

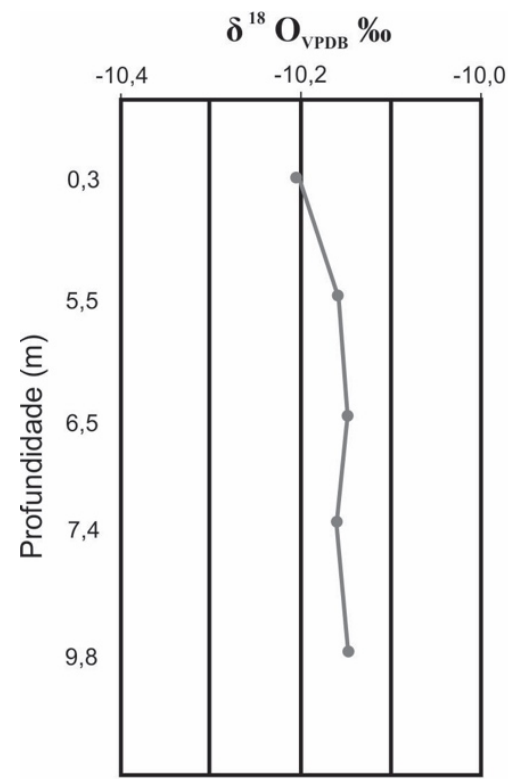

A

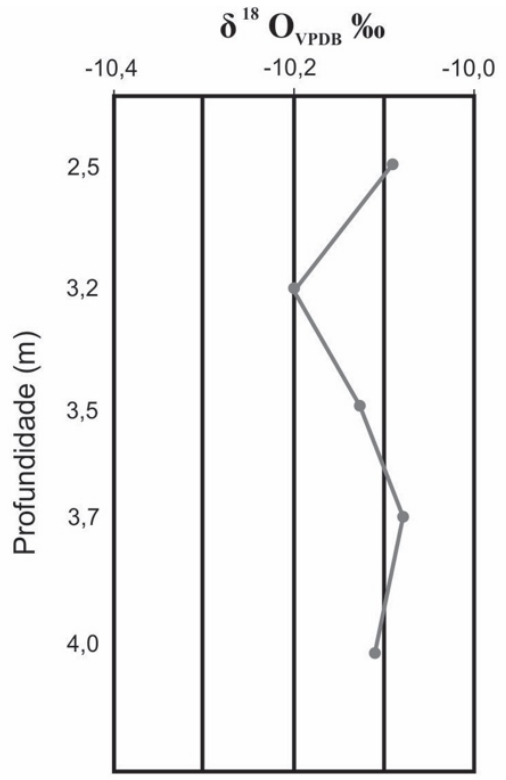

B

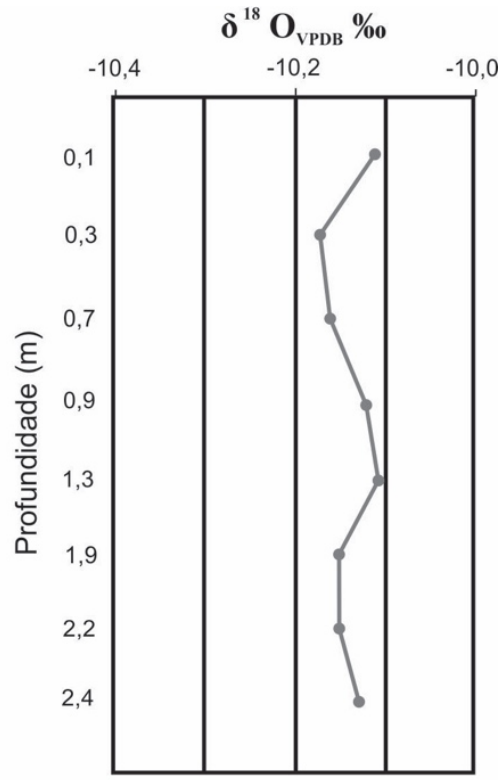

C

Figura 2

Distribuição de $\delta^{18} \mathrm{O}$ em carapaças de ostracodes em carapaças de ostracodes ao longo das seções (A), Arrojado, (B) Canastra e (C) Cedro.

Tais valores estão dentro da média global de calcários marinhos para o Cretáceo, e apresentam características típicas da época, demonstrando que não havia grande variação de temperatura da água, e estas permaneciam altas como em períodos interglaciais. Em 
relação a salinidade, as amostras estudadas, também estão dentro da média global para o Cretáceo, cujo os valores de calcários marinhos variam entre $-30 \%$ e $-4 \%$ (Anderson \& Arthur, 1983) (Figura 3).
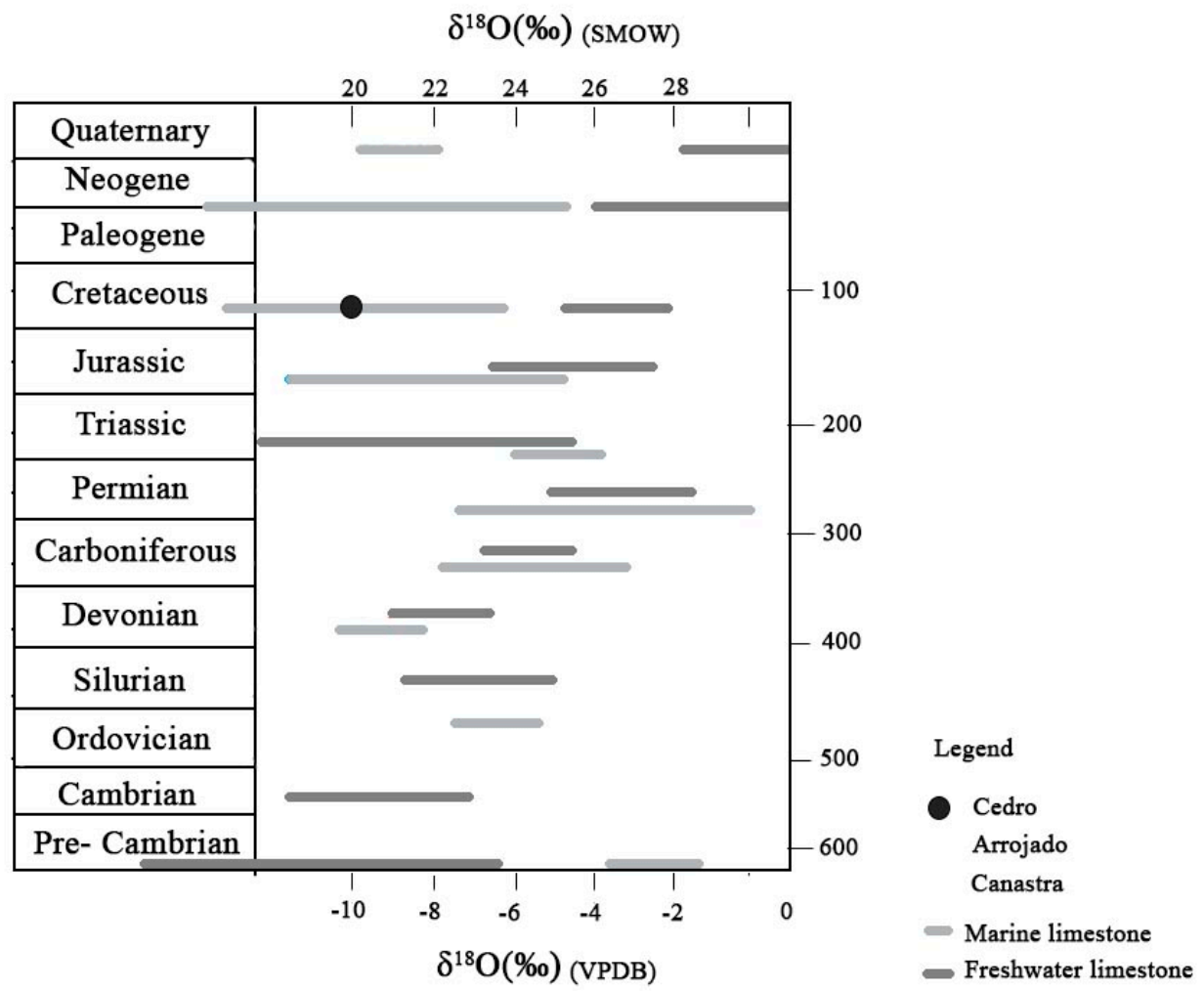

Figura 3 Variação de isótopos de oxigênio de cherts e carbonatos com o tempo geológico (Modificado de Anderson \& Arthur, 1983).

\subsection{ANÁLISE DE $\delta^{13} \mathrm{C}$}

Na figura 4 é possível observar os valores da composição isotópica do $\mathrm{C}^{13}$ dos afloramentos estudados. $\mathrm{O}$ afloramento Arrojado teve valores que oscilaram entre -
$7,6 \%$ e $-2,7 \%$. Os valores do afloramento Canastra oscilaram entre $-12,6 \%$ e $-4,5 \%$ e os valores do afloramento Cedro oscilaram entre $4,7 \%$ e $+2,7 \%$.

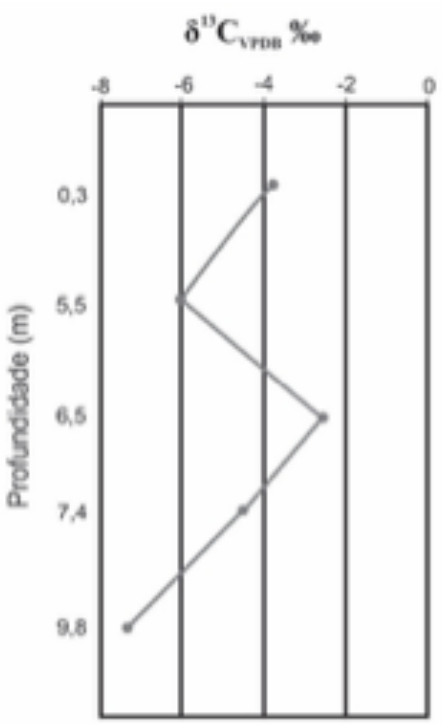

A

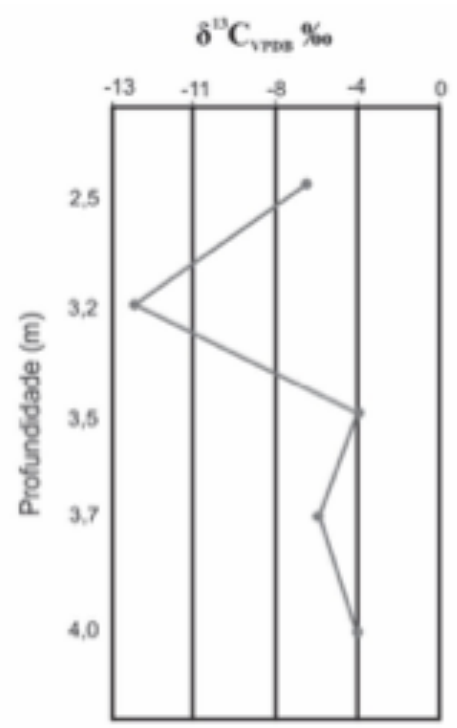

B

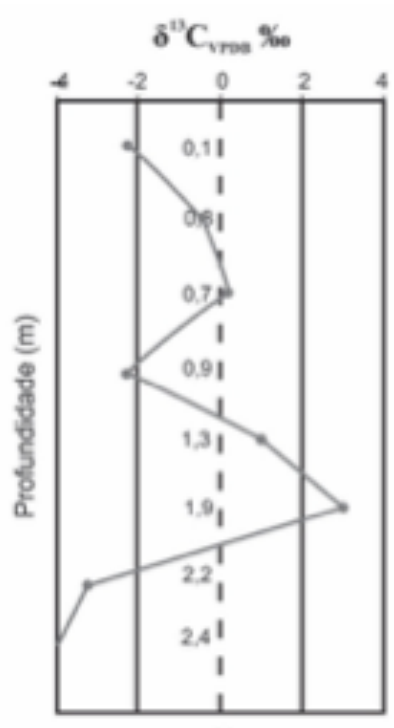

C

Figura 4

Distribuição de $\delta^{13} \mathrm{C}$ em carapaças de ostracodes ao longo dos afloramentos (A) Arrojado, (B) Canastra e (C) Cedro. 
Os afloramentos Arrojado e Canastra apresentaram apenas valores negativos oscilando ao longo das seções, indicando um rápido soterramento de matéria orgânica deixando a água empobrecida em $\mathrm{C}^{13}$ provavelmente resultado de um ambiente anóxico.

Por se tratar de afloramentos com caráter transgressivo, os valores mais negativos são esperados. Segundo Rodrigues \& Fauth (2013), em episódios de transgressões marinhas e inundações de plataformas carbonáticas, são registradas as melhores condições para preservação da matéria orgânica. Desta forma, durante períodos de anoxia os folhelhos de ambientes marinhos apresentam valores da razão de $\delta^{13} \mathrm{C}$ mais negativos. Este empobrecimento se deve à alta concentração de $\mathrm{C}^{12}$ no ambiente.

A associação de microfósseis encontrada nesses afloramentos é bastante semelhante, com a ocorrência de espécies de ostracodes mixohialinos em abundância na base dos afloramentos e, a ocorrência de foraminíferos bentônicos no topo (Figura 5).
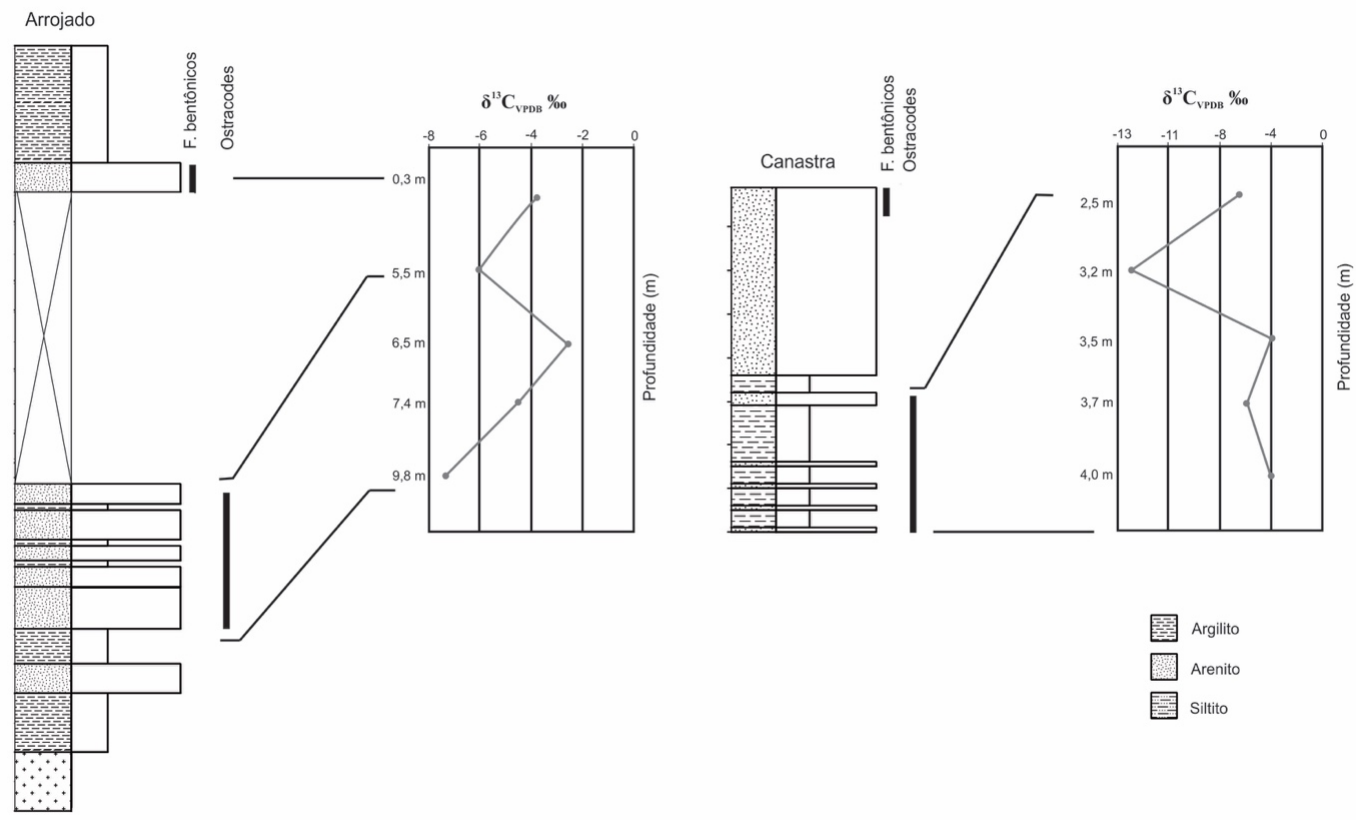

Figura 5

Perfis estratigráficos dos Afloramentos Arrojado e Canastra; Correlação dos microfóssies com os dados de $\delta^{13} \mathrm{C}$ em carapaças de ostracodes.

O afloramento Cedro (Figura 6) foi o único que oscilou entre valores positivos e negativos ao longo do perfil estratigráfico. No meio da seção é possível observar um aumento súbito de $-4,2 \%$ para $+2,7 \%$, seguido por uma queda brusca de $+1,5 \%$ para $-2,2 \%$ e logo após por um gradual decaimento no topo da seção. De acordo com Berger e Vincent (1986) a remoção de $1 \%$ do reservatório oceânico de carbono orgânico produz 0,2 \%o de mudança positiva. Os valores positivos observados nas amostras intermediárias do afloramento Cedro tem uma amplitude de $6,9 \%$, o que indica uma mudança significativa no fluxo de carbono durante esse intervalo. Por se tratar de espécies de ostracodes epifaunais, os valores de $\mathrm{C}^{13}$ são mais influenciados pelas variações do ambiente que pelo próprio micro-habitat (Decrouy et al. 2011).
Segundo Hoefs (2004) e Armstrong \& Brasier (2005), os carbonatos marinhos são isotopicamente mais pesados e apresentam um valor médio de razão de $\delta^{13} \mathrm{C}$ ao redor de $0 \%$ (variável entre 1 e $3 \%$ ). Nesse estudo, os valores mais positivos nos níveis 1,90 e 1,30 m indicam que a deposição provavelmente ocorreu em ambiente com maior influência marinha, os carbonatos de ambientes marinhos tendem a possuir valores de $\delta^{13} \mathrm{C}$ mais positivos em períodos de alta produtividade orgânica (Rodrigues e Fauth, 2013).

Esse dado é corroborado pela associação de microfósseis, onde é registrada uma maior diversidade de foraminíferos (com presença de foraminíferos planctônicos) e ostracodes mixohialinos que estariam associados a um ambiente marinho nesses intervalos. 

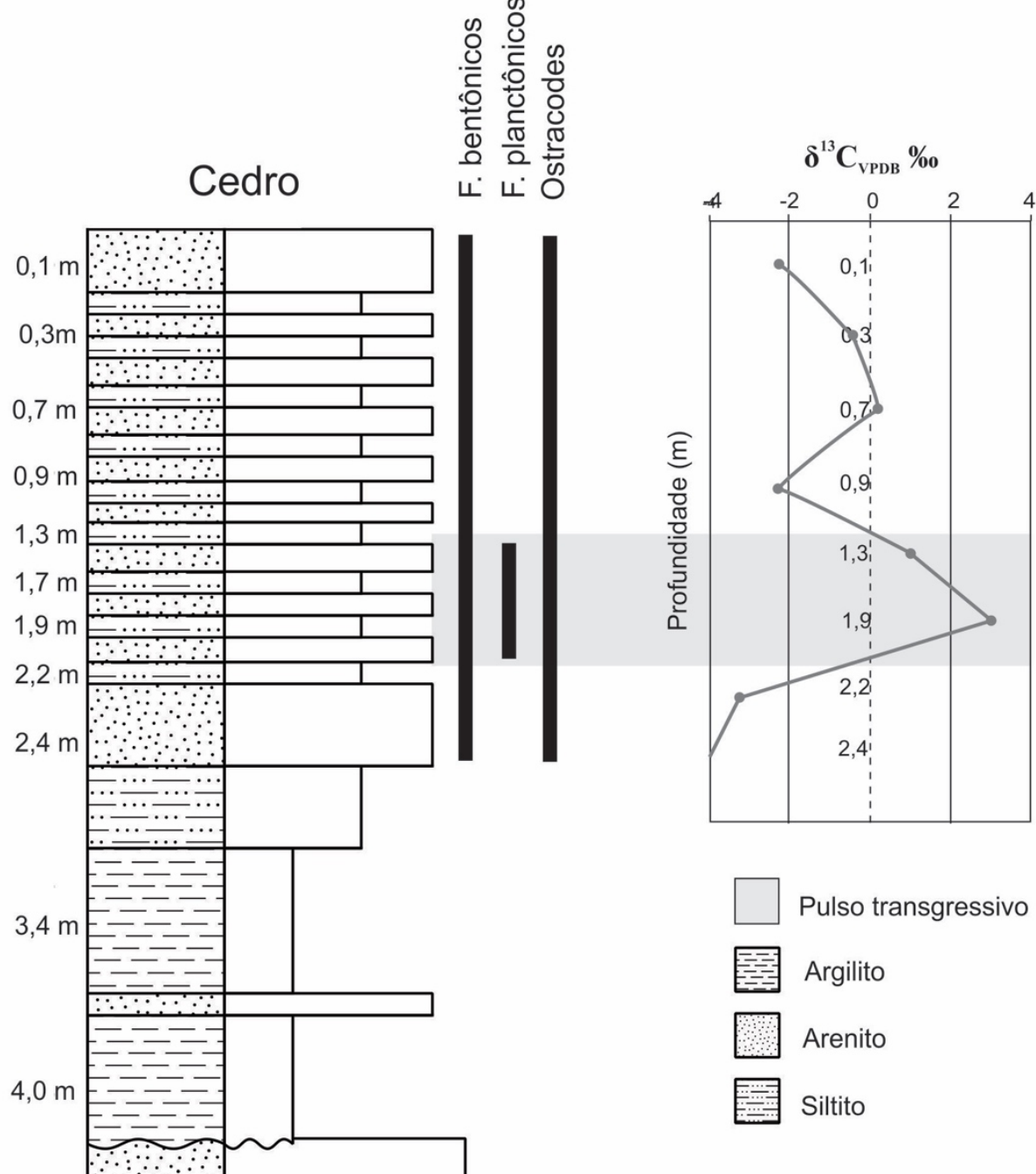

Arenito
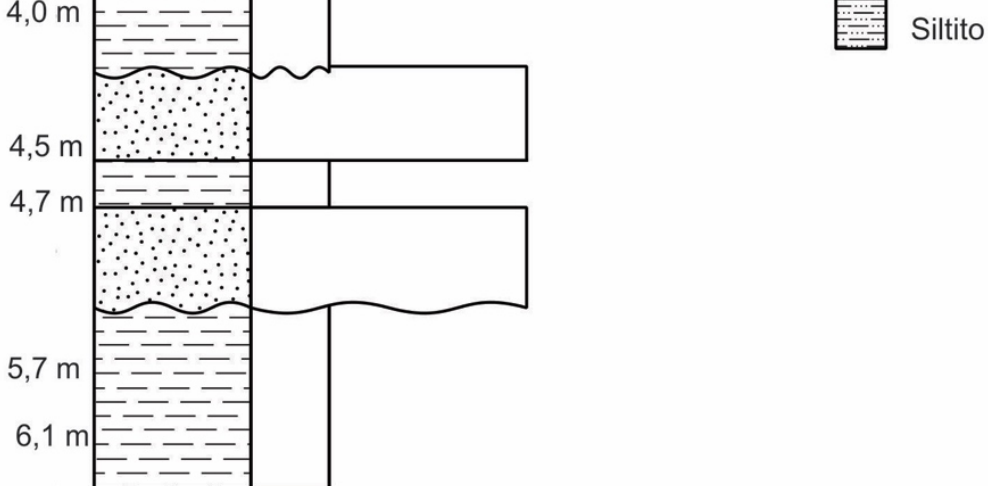

Figura 6

Perfil estratigráfico do afloramento Cedro (6,1 metros), Correlação dos microfóssies com os dados de $\delta^{13} \mathrm{C}$ em carapaças de ostracodes caracterizando um pulso transgressivo entre 2,2 m e 1,3 $\mathrm{m}$ de profundidade da seção analisada.

Os dados isotópicos de carbono indicam uma maior similaridade entre os afloramentos Arrojado e Canastra, que se localizam na porção sudoeste da bacia. Porém, no afloramento Cedro, que se localiza na porção centro-sul, é possível observar que os valores isotópicos de carbono tendem a ser mais positivos em determinados níveis da seção, demonstrando que a região centro-sul sofreu uma maior influência marinha do que a região sudoeste da Bacia do Araripe. 


\section{CONCLUSÃO}

Os dados isotópicos de oxigênio de uma maneira geral se apresentam bastante similares, nos três afloramentos, com valores negativos e com pouca variação indicando um ambiente de águas com temperaturas elevadas e precipitação de calcários marinhos nos afloramentos.

Os dados isotópicos de carbono nos três afloramentos (Canastra, Arrojado e Cedro) indicaram ambiente de caráter trangressivo, sugerindo ambiente anóxico e com alto teor de matéria orgânica. Porém a variação positiva

\section{AGRADECIMENTOS}

À Coordenação de Aperfeiçoamento de Pessoal de Nível Superior (CAPES) pela bolsa de mestrado concedida para a primeira autora. Ao Conselho Nacional de Desenvolvimento

\section{REFERÊNCIAS}

Allan, J.R., Matthews, R.K., 1982, Isotope signatures associated with earlymeteoric diagenesis: Sedimentology, 29, 797-817.

Anderson, T.F., Arthur, M.A., 1983, Stable isotopes of oxygen and carbono and their application to sedimentologic and paleoenvironmental problems, in Arthur, M.A.,

Anderson, T.F., Kaplan, I.R., Veizer, J., Land, L.S. (eds.), Stable Isotopes in Sedimentary Geology: Society of Economic Paleontologists and Mineralogists, Short Course Notes., 10, 1-151.

Armstrong, H.A., Brasier, M.D. 2005. Microfossil, stable isotopes and ocean atmosphere history. In: Microfossils. Blackwell Publishing Malden-USA. 2 ed., 4:25 34.

Armstrong-Altrin, J.S., Madhavaraju, J., Sial, A.N., Kasper-Zubillaga, J.J., Nagarajan, R., FloresCastro, K., Rodríguez, J.L., 2011, Petrography and stable isotope geochemistry of the Cretaceous El Abra Limestones (Actopan), Mexico: Implication on diagenesis: Journal of the Geological Society of India., 77(4), 349359.

Assine, M. L. 2007. Bacia do Araripe. Boletim de Geociências da Petrobrás., 15(2):371-389.

Assine, M. L. 1992. Análise Estratigráfica da Bacia do Araripe, Nordeste do Brasil. Revista Brasileira De Geociências,v.22, n. 3, p.289300.

Assine, M. L., Perinotto, J. A. J., Custódio, M. A., Neumann, V. H., Varejão, F. G. e Mescolotti, P. C. 2014. Sequências deposicionais do Andar Alagoas da Bacia do Araripe, Nordeste do Brasil. Boletim de Geociências da Petrobras, Rio de Janeiro, v. 22, n. 1, p. 3-28. registrada no afloramento Cedro, juntamente com associação de microfósseis carbonáticos, indica alteração no fluxo de carbono ocasionada pela oscilação no ambiente marinho.

De acordo com a análise isotópica e a análise da associação de microfósseis é possível inferir que o ambiente deposicional do afloramento Cedro tem características ligadas a um ambiente com maior influência marinha do que nos afloramentos Arrojado e Canastra.

Científico e Tecnológico (CNPq) pelo suporte financeiro (processos 40714812010-3, 201716/2010-0 e 303071/2014-1).

Berger, W.H., Vincent, E., 1986, Deep sea carbonates: reading the carbono isotope signal: Geologische Rundschau, 75, 249-269.

Camoin, G., Casanova, J., Rouchy, J. M., BlancValeron, M. M., e Deconinck, J. F., 1997. Environmental control son perennial and ephemeral carbonate lakes: the central PalaioAndian basin of Bolivia during Late Cretaceous to Early Tertiary times. Sedimentary Geology, 113, p. 1-26.

Decrouy L., Vennemann T. W., Ariztegui D. 2011. Controls on ostracod valve geochemistry, Part 1: Variations of environmental parameters in ostracod (micro-)habitats. Geochimica et Cosmochimica Acta 75 (2011) 7364-7379.

Hoefs J. 2004. Stable Isotope in Geochemistry. Springer- Verlag. Germany. 5 ed. 244 p.

Holmes, J.A. J., 1996. Trace-element and stableisotope geochemistry of non-marine ostracod shells in Quaternary palaeoenvironmental reconstruction Paleolimnol. 15: 223. https://doi.org/10.1007/BF00213042

Kaufman, A.J., Knoll, A.H., 1995, Neoproterozoic variations in the $\mathrm{C}$-isotopic composition of seawater: stratigraphic and biogeochemical implications: Precambrian Research, 73(1-4), 27-49.

Leckie, R. M. e Olson, H. C. 2012. Foraminifera as proxies for sea-level change on siliciclastic margins. (SEMP) Society for sedimentary Geology, ISBN 1 56576- 084-0, p. 5-19.

Leipnitz, I.I. \& Aguiar, E.S. 2002. Foraminíferos Recentes e Fósseis. in:t.l. dutra (org.); técnicas e procedimentos de trabalho com fósseis e formas modernas comparativas. São Leopoldo, ppgeo/unisinos/capes. $\mathrm{n}^{\mathrm{o}} 01$. editora unisinos. $\mathrm{p}$. 8-10.

Geochimica Brasiliensis 33(2): 133 - 142, 2019 
Madhavaraju J., Sial A.N., González-León C.M. \& Nagarajan R. 2013b. Carbon and oxygen isotopic variations in Early Albian limestone facies of the Mural Formation, Pitaycachi section, northeastern Sonora, Mexico.- Revista Mexicana de Ciencias Geologicas, Mexico City, vol. 30, p. 526-539.

Madhavaraju, J., Kolosov, I., Buhlak, D., Armstrong-Altrin, J.S., Ramasamy, S., Mohan, S.P., 2004, Carbon and oxygen isotopic signatures in Albian-Danian limestones of Cauvery basin, southeastern India: Gondwana Research, 7(2), 527-537.

Marshall, J.D., 1992, Climatic and oceanographic isotopic signals from the carbonate rock record and their preservation: Geological Magazine, 129, 143-160.

Pearson P. N. 2012. Oxygen Isotopes In Foraminifera: Overview And Historical Review, In: Ivany, L.C., Huber, B.T. (Eds.), Reconstructing Earth's Deep-Time ClimateThe State of the Art in 2012. The Paleontological Society, p.: 1-38

Ponte, F.C. 1992. Origem e evolução das pequenas bacias cretácicas do interior do Nordeste do Brasil. In:Simpósio sobre as Bacias Cretácicas.

Rodrigues, G. B.; Fauth, G. 2013 Isótopos estáveis de carbono e oxigênio em ostracodes do Cretáceo: metodologias, aplicações e desafios. Terrae Didática, v. 9, p. 34-49.
Spero H. J., Bijma J., Lea D.W., Bemis B.E. 1997. Effect of seawater carbonate concentration on foraminiferal carbon and oxygen isotopes. Nature, 390:497-500

Talbot, M. R. \& K. R. Kelts (eds), 1989. Phanerozoic record of lacustrine basins and their environmental signals. Palaeogeog. Palaeoclimat. Palaeoecol. 70: 1-304.

Talbot, M. R., 1990. A review of the paleohydrological interpretation of carbon and oxygen isotope ratios in primary lacustrine carbonates. Chemical Geology (Isoptope Geoscience Section), 80, p. 261-279.

Veizer, J., 1983, Chemical diagenesis of carbonates; theory and application of trace element technique, in Arthur, M.A., Anderson, T.F., Kaplan, I.R., Veizer, J., Land, L.S. (eds.), Stable Isotopes in Sedimentary Geology: Society of Economic Palaeontologists and Mineralogists, Short Course 10, 3-100.

Viana, M.S.S.; Neumann, V.H.L., 2002. Membro Crato da Formação Santana, Chapada do Araripe, CE: riquíssimo registro de fauna e flora do Cretáceo. In: Schobbenhaus, C.; Campos, D.A.; Queiroz, E.T., Winge, M.; Berbert-Born, M.L.C. (eds.) Sítios Geológicos e Paleontológicos do Brasil. DNPM/CPRM Comissão Brasileira de Sítios Geológicos e Paleobiológicos (SIGEP) - Vol. I, p. 113-120. 